\title{
Dima Khavinson's 60th: A conference on complex functions, operators, partial differential equations, and applications in mathematical physics
}

\author{
Erik Lundberg ${ }^{1} \cdot$ Razvan Teodorescu $^{2}$
}

Published online: 30 May 2018

(C) Springer International Publishing AG, part of Springer Nature 2018

\section{Foreword}

This special issue of Analysis and Mathematical Physics is based on presentations given during the conference dedicated to Dmitry (Dima) Khavinson's 60th anniversary, held in June 2017 at the Mittag Leffler Institute, Stockholm. The general theme of this event was focused on those areas in which Professor Khavinson has made significant contributions, such as obtaining a number of fundamental results in several fields of mathematical analysis, and creating novel connections between subjects and methods as different as pure complex analysis and applied astrophysics.

The special issue consists of original papers pertaining to these research areas from the field of mathematical analysis in general, and in function theory, partial differential equations, harmonic analysis and potential theory, in particular. These are complemented by a selection of open problems, proposed by some of the conference participants, organized thematically.

Dima Khavinson's work with Harold Shapiro in the 1980s on several free boundary problems is nowadays credited with creating an entire branch of research in complex analysis and mathematical physics, and is referenced repeatedly in the considerable literature on Laplacian Growth processes. Central objects in this field are the Schwarz function of the boundary of a domain (in two dimensions), and the Schwarz potential (in general). These are tightly related to inverse moment problems and inverse potential problems, both in the sense of approximation theory and in harmonic analysis. The articles "Line bundles defined by the Schwarz function" and "On moments of a

\footnotetext{
$凶 \quad$ Razvan Teodorescu razvan@usf.edu

1 Department of Mathematics and Statistics, Florida Atlantic University, Boca Raton, FL, USA

2 Department of Mathematics and Statistics, University of South Florida, Tampa, FL, USA
} 
polytope" are representative for this (vast) field of research, and relate naturally to the open problems "The Schwarz potential and curvature", "Sharp upper bounds for the curvature of some free boundaries in higher dimensions", "The classification problem for arclength null-quadrature domains", and "A free boundary problem for conformal maps arising in random aggregation" from the concluding section.

Together with Harold Shapiro and Peter Duren, Dima Khavinson's work on function theory in Bergman spaces, in the early 1990s, is considered nowadays a canonical, fundamental contribution to that entire field, influencing the development of that subject for decades to come. His article (with Peter Ebenfeld) in 1997 on the extension of the Schwarz reflection principle to functions of several complex variables completely answered a famous problem that had been around for over a century and whose solution had evaded the best analysts in the world. This volume's articles "Extension results for harmonic functions which vanish on cylindrical surfaces" and "The adjoint of a composition operator via its action on the Szegö kernel", as well as the open problem "Backward uniqueness for the heat equation" point to the diversity of questions pursued and methods used in this well-developed field of research.

Similarly, Dr. Khavinson's results on zeros of harmonic polynomials and rational functions, and their subsequent applications to gravitational lensing in astrophysics, not only settled an outstanding open problem with a classical flavor, but also opened new directions in this vast area of science. The flurry of activity these discoveries prompted both in pure mathematics and in theoretical (as well as experimental) astrophysics would indeed deserve a separate, specialized volume. This issues' articles "On the number of solutions of some transcendental equations" and "On the zeros of random harmonic polynomials: the Weyl model", together with the open problem "Equilibrium points of logarithmic and Newtonian potentials", give some perspective on this very active area of research.

No survey of Dima Khavinson's work would be complete without a section dedicated to his work on invariant subspace problems and generalizations of the notion of inner function. For mathematical physics, these relate to the very foundation of quantum theory: finding the subspace of "physical states" in a larger (unphysical) Hilbert space, and in particular finding a "vacuum" vector (a cyclic vector solving an optimization problem) with the proper boundary behavior. This volume's article "A note on cyclic polynomials in polydiscs" and open problems "Zeros of weighted reproducing kernels", "Inner functions in $H^{2}$ ", and "Inner functions in $H_{\omega}^{2}$ " offer a glimpse of the beauty and difficulty of this (rapidly expanding) field of research.

We invite the reader to enjoy the celebration of the many areas and aspects of mathematical research offered in the present volume.

May 2018

Erik Lundberg and Razvan Teodorescu 